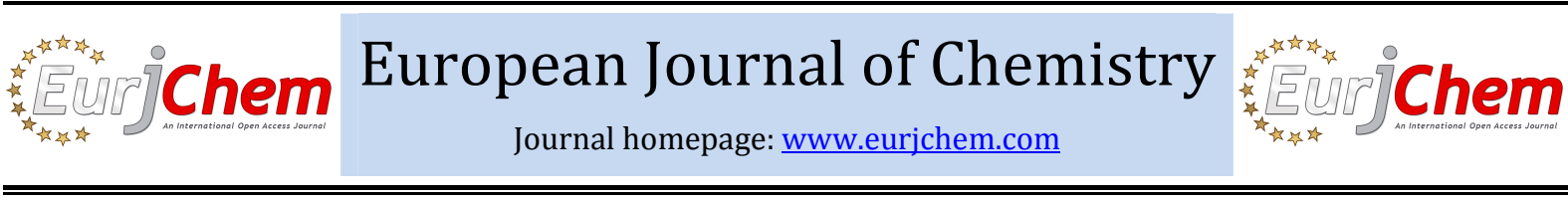

\section{Synthesis and spectral characterization of some heterocyclic nitrogen compounds}

\author{
Mamdouh Adly Hassan a, Maghrabi Ali Seleem b, Ahmed Mohamed Mosallem Younes b, \\ Mohamed Mobark Taha ${ }^{\mathrm{b}}$ and Abou-Bakr Haredi Abdel-Monsef $\mathrm{b}$, * \\ a Pharmaceutical Chemistry Department, Faculty of Pharmacy, Sinai University, Arish, 45518, Egypt \\ ${ }^{\mathrm{b}}$ Chemistry Department, Faculty of Science, South Valley University, Qena, 83523, Egypt \\ ${ }^{*}$ Corresponding author at: Chemistry Department, Faculty of Science, South Valley University, Qena, 83523, Egypt. \\ Tel.: +20.96.5211281; fax: +20.96.5211279. E-mail address: bakooos2004@yahoo.com (A.H. Abdel-Monsef).
}

\section{ARTICLE INFORMATION}

Received: 04 February 2013

Accepted: 16 February 2013

Online: 30 June 2013

\section{KEYWORDS}

Arylidine

Hydrazide

Pyrimidines

Carbon disulphide

Quinazolin-2,4-dione

Glycine ethyl ester hydrochloride

\section{Introduction}

It is well known that the quinazolines and pyrimidines derivatives are compounds with high biological activities. As an important class of electron-rich $\mathrm{N}$-containing heterocycles, pyrimidines are widely present in biologically active compounds and have also versatile synthetic applications. Many derivatives of pyrimidines showed antifungal [1], antibacterial [2], anti-inflammatory [3] anticonvulsant [4-6]. Pyrimidines [7] encourage the authors to gather these moieties hoping to produce valuable new compounds of expected antibacterial and antifungal activity. We report here the synthesis of novel quinazolin-2,4-dione derivatives starting from (2,4-dioxo-1,4-dihydro-2H-quinazolin-3-yl)-acetic acid hydrazide (3). All the synthesized compounds have been supported by their spectral data.

\section{Experimental}

\subsection{Instrumentation}

Melting points were uncorrected and determined on an electric melting point apparatus (Kofler). The IR spectra (KBr) were recorded on a Shimadzu 408 spectrometer. The ${ }^{1} \mathrm{H}$ NMR spectra were recorded using $300 \mathrm{MHz}$ Varian EM 390 spectrometer; chemical shifts are reported in ppm with TMS as an internal standard and are given in $\delta$ units. Electron impact mass spectra were obtained at $70 \mathrm{eV}$ with Shimadzu GC-MS (QP-2010 plus). Elemental analyses were carried out at the Microanalysis Unit at Cairo University. The purity of the compounds was detected by TLC.

\subsection{Synthesis}

2.2.1. (2,4-Dioxo-1,4-dihydro-2H-quinazolin-3-yl)-acetic acid ethyl ester (2)

A mixture of $N$-phenylsulphonyloxyphthalimide (1) $(5 \mathrm{~g}$, $0.016 \mathrm{~mol}$ ) and glycine ethyl ester hydrochloride $(2.99 \mathrm{~g}, 0.02$ mol) in pyridine $(20 \mathrm{~mL})$ was refluxed for 9 hours. After cooling; the reaction mixture was acidified with cold dilute hydrochloric acid (1:1), and the solid formed was filtered off and dried. The target product was crystallized from benzene to give compound $\mathbf{2}$ as a gray crystal (Scheme 1). Yield: $3.7 \mathrm{~g}$, 90\%. M.p.: 206-208 ${ }^{\circ} \mathrm{C}$. FT-IR (KBr, $\left.v, \mathrm{~cm}^{-1}\right): 3380$ (NH), 1719, 1671 (C=0's). ${ }^{1} \mathrm{H}$ NMR (300 MHz, DMSO- $\left.d_{6}, \delta, \mathrm{ppm}\right): 1.1(\mathrm{t}, 3 \mathrm{H}$, $\left.\mathrm{CH}_{3}\right), 4.1$ (q, 2H, $\mathrm{CH}_{2}$ ), 4.6 (s, $\left.2 \mathrm{H}, \mathrm{N}-\mathrm{CH}_{2}\right), 7.2-7.9$ (m, $4 \mathrm{H}$, arom.), $11.6(\mathrm{~s}, 1 \mathrm{H}, \mathrm{NH})$. MS ( $\mathrm{m} / \mathrm{z}, \%): 248(31.1 \%)\left(\mathrm{M}^{+}\right)$. Anal. calcd. for $\mathrm{C}_{12} \mathrm{H}_{12} \mathrm{~N}_{2} \mathrm{O}_{4}$ : C, 58.06; H, 4.87; N, 11.28. Found: C, 58.25; $\mathrm{H}$, $4.89 ; \mathrm{N}, 11.35 \%$.

\subsection{2. (2,4-Dioxo-1,4-dihydro-2H-quinazolin-3-yl)-acetic acid hydrazide (3)}

To a solution of compound $2(2.5 \mathrm{~g}, 0.01 \mathrm{~mol})$ in absolute ethanol $(30 \mathrm{~mL}), 5 \mathrm{~mL}$ of hydrazine hydrate was added; the reaction mixture was refluxed for 8 hours. The reaction mixture was allowed to cool and the separated product was filtered and dried. Crystallization of the crude product with ethanol and acetic acid, afforded compound $\mathbf{3}$ as a white crystal (Scheme 1). Yield $1.6 \mathrm{~g}, 62$ \%. M.p.: >300. FT-IR $\left(\mathrm{KBr}, v, \mathrm{~cm}^{-1}\right)$ : $3296(\mathrm{NH}), 3196\left(\mathrm{NH}_{2}\right), 1714,1665\left(\mathrm{C}=\mathrm{O}^{\prime} \mathrm{s}\right) .{ }^{1} \mathrm{H}$ NMR $(300$ MHz, DMSO- $\left.d_{6}, \delta, \mathrm{ppm}\right): 4.5\left(\mathrm{~s}, 2 \mathrm{H}, \mathrm{CH}_{2}\right), 4.2\left(\mathrm{~s}, 2 \mathrm{H}, \mathrm{NH}_{2}\right), 7.1-$ 7.9 (m, 4H, arom.), 9.2 (s, 1H, NH), $11.5(\mathrm{~s}, 1 \mathrm{H}, \mathrm{NH})$. 


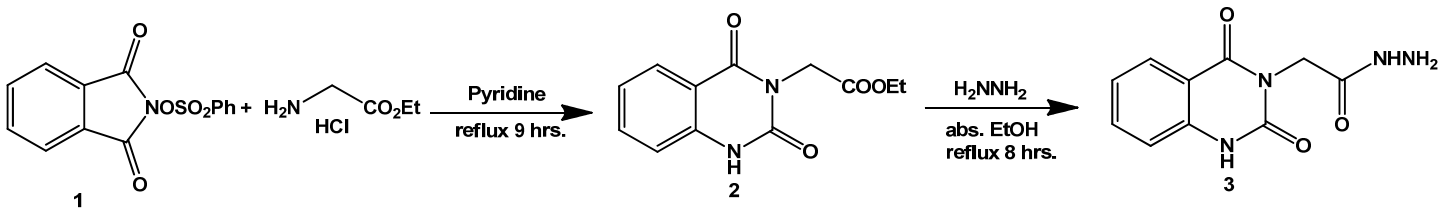

Scheme 1

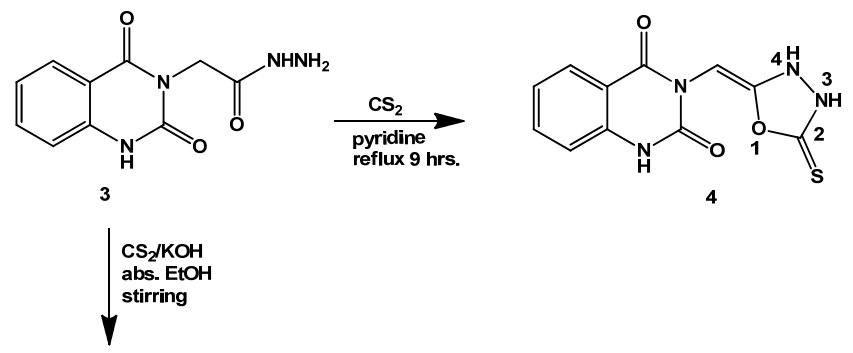<smiles>Cc1cc2[nH]c(=O)n(CC(=O)NNC(=S)[SiH2]c3cccc(N)c3)c(=O)c2c(=O)n1Cc1nnc(S)n1N</smiles>

Scheme 2

MS (m/z, \%): $234(20.1 \%)\left(\mathrm{M}^{+}\right)$. Anal. calcd. for $\mathrm{C}_{10} \mathrm{H}_{10} \mathrm{~N}_{4} \mathrm{O}_{3}$ : C, 51.28; H, 4.30; N, 23.92. Found: C, 51.49; H, 4.31; N, 23.98\%.

\subsubsection{3-(5-Thioxo-[1,3,4]oxadiazolidin-2-ylidenemethyl)-1H- quinazoline-2,4-dione (4)}

To a solution of compound $3(0.5 \mathrm{~g}, 0.002 \mathrm{~mol})$ in pyridine (20 mL), $1 \mathrm{~mL}$ of carbon disulphide was added; the reaction mixture was refluxed for 10 hours or until the hydrogen sulfide has been evolved. After cooling; the reaction mixture was acidified with cold dilute hydrochloric acid (1:1), and the solid formed was filtered off and dried. The product was crystallized from acetic acid to give compound $\mathbf{4}$, as a yellowish white crystal (Scheme 2). Yield 0.3 g, 60 \%. M.p.: $274{ }^{\circ} \mathrm{C} .{ }^{1} \mathrm{H}$ NMR $(300$ MHz, DMSO- $\left.d_{6}, \delta, \mathrm{ppm}\right): 4.6(\mathrm{~s}, 1 \mathrm{H}$, Olifinic $\mathrm{CH}), 5.1(\mathrm{~s}, 1 \mathrm{H}$, $\mathrm{NH}(4)$ ), 7.2-7.9 (m, 4H, arom.), 10.2 (s, 1H, NHCS), 11.5 (s, 1H, $\mathrm{NH}$ ). Anal. calcd. for $\mathrm{C}_{11} \mathrm{H}_{8} \mathrm{~N}_{4} \mathrm{O}_{3} \mathrm{~S}$ : C,47.82; $\mathrm{H}, 2.92 ; \mathrm{N}, 20.28$; $\mathrm{S}$, 11.61. Found: C, 48.02; H, 2.94; N, 20.35; S, 11.59\%.

\subsubsection{3-(4-Amino-5-mercapto-4H-[1,2,4]triazol-3-ylmethyl)- 1H-quinazoline-2,4-dione (5)}

To a solution of (2,4-dioxo-1,4-dihydro- $2 \mathrm{H}$-quinazolin-3yl)-acetic acid hydrazide (3) (1 g, $0.004 \mathrm{~mol}$ ) in absolute ethanol $(30 \mathrm{~mL})$, potassium hydroxide $(0.45 \mathrm{~g}, 0.008 \mathrm{~mol})$ and carbon disulfide $(3 \mathrm{~mL})$ were added, respectively. The mixture was stirred for 8 hours in ice bath. The yellow formed salt was filtered off and dried. Then the solid was dissolved in distilled water $(50 \mathrm{~mL})$ and hydrazine hydrate $(0.1 \mathrm{~mol})$ was added to this solution with reflux for 10 hours, after that the mixture was neutralized with diluted acetic acid to form white precipitate which collected by filtration. The resulting product was recrystallized from ethanol to give compound $\mathbf{5}$ as a white crystal (Scheme 2). Yield $0.8 \mathrm{~g}, 66 \%$. M.p.: $282^{\circ} \mathrm{C}$. FT-IR (KBr, $v$, $\mathrm{cm}^{-1}$ ): $3304(\mathrm{NH}), 3203,3169\left(\mathrm{NH}_{2}\right), 1715,1660$ (C=O' s). ${ }^{1} \mathrm{H}$ NMR (300 MHz, DMSO- $\left.d_{6}, \delta, \mathrm{ppm}\right): 5.1\left(\mathrm{~s}, 2 \mathrm{H}, \mathrm{NH}_{2}\right), 5.7(\mathrm{~s}, 2 \mathrm{H}$, $\mathrm{CH}_{2}$ ), 7.2-7.9 (m, 4H, arom.), 11.6 (s, $\left.1 \mathrm{H}, \mathrm{NH}\right), 13.5$ (s, $\left.1 \mathrm{H}, \mathrm{SH}\right)$. Anal. calcd. for $\mathrm{C}_{11} \mathrm{H}_{10} \mathrm{~N}_{6} \mathrm{O}_{2} \mathrm{~S}: \mathrm{C}, 45.51 ; \mathrm{H}, 3.47 ; \mathrm{N}, 28.95 ; \mathrm{S}, 11.04$. Found: C, 45.80; H, 3.49; N, 29.02; S, 11.24\%.

\subsubsection{General procedures for synthesis of arylidines (6a-d)}

(2,4-Dioxo-1,4-dihydro-2H-quinazolin-3-yl)-acetic acid hydrazide $3(0.45 \mathrm{~g}, 0.002 \mathrm{~mol})$ was heated under reflux for 10 12 hours with the appropriate aromatic aldehydes namely benzaldehyde, $p$-nitrobenzaldehyde, 2-furaldehyde and thiophenecarbaldehyde $(0.003 \mathrm{~mol})$ in absolute ethanol $(20$ $\mathrm{mL}$ ) and in presence of piperidine as a catalyst. After cooling; the reaction mixture was filtered off and crystallized from appropriate solvent to give the arylidine derivatives 6a-d, respectively (Scheme 3).

(2,4-Dioxo-1,4-dihydro-2H-quinazolin-3-yl)-acetic acid benzylidene hydrazide (6a): White crystal. Yield: $0.3 \mathrm{~g}, 55 \%$. M.p.: > $300{ }^{\circ} \mathrm{C}$. FT-IR (KBr, v, cm$\left.{ }^{-1}\right): 3258(\mathrm{NH}), 3102(\mathrm{CH}$ aliphatic), 1722, 1693 (C=0' s). ${ }^{1} \mathrm{H}$ NMR (300 MHz, DMSO- $d_{6}, \delta$, ppm): 5.0 (s, $\left.2 \mathrm{H}, \mathrm{CH}_{2}\right), 7.2-8.2(\mathrm{~m}, 9 \mathrm{H}$, arom.), $8.01(\mathrm{~s}, 1 \mathrm{H}, \mathrm{CH})$, 11.54 (s, 1H, NH), 11.65 (s, 1H, NH). MS ( $\mathrm{m} / \mathrm{z}, \%): 322$ (2.4 \%). Anal. calcd. for $\mathrm{C}_{17} \mathrm{H}_{14} \mathrm{~N}_{4} \mathrm{O}_{3}$ : C, 63.35; H, 4.38; N, 17.39. Found: C, 63.53; H, 4.41; N, 17.45\%.

(2,4-Dioxo-1,4-dihydro-2H-quinazolin-3-yl)-acetic acid (4nitrobenzylidene)-hydrazide (6b): White crystal .Yield $0.34 \mathrm{~g}$, 56\%. M.p.: > $300{ }^{\circ} \mathrm{C}$. FT-IR (KBr, $\left.v, \mathrm{~cm}^{-1}\right): 3206(\mathrm{NH}), 3183(\mathrm{CH}$ aliphatic), 1715, 1694 (C=0' s). ${ }^{1} \mathrm{H}$ NMR (300 MHz, DMSO-d $d_{6}, \delta$, ppm): 5.0 (s, $2 \mathrm{H}, \mathrm{CH}_{2}$ ), 7.2-8.3 (m, $8 \mathrm{H}$, arom.), 8.0 (s, $\left.1 \mathrm{H}, \mathrm{CH}\right)$, 11.6 (s, 1H, NH), $12.0(\mathrm{~s}, 1 \mathrm{H}, \mathrm{NH})$. MS ( $\mathrm{m} / \mathrm{z}, \%): 367$ (3.0\%). Anal. calcd. for $\mathrm{C}_{17} \mathrm{H}_{13} \mathrm{~N}_{5} \mathrm{O}_{5}$ : C,55.58; $\mathrm{H}, 3.56 ; \mathrm{N}, 19.04$. Found: C, $55.60 ; \mathrm{H}, 3.58 ; \mathrm{N}, 19.02 \%$.

(2,4-Dioxo-1,4-dihydro-2H-quinazolin-3-yl)-acetic acid (furan-2-ylmethylene)-hydrazide (6c): Yellow crystal. Yield: 0.4 g, 72\%. M.p.: > $300{ }^{\circ} \mathrm{C}$. FT-IR (KBr, v, cm$\left.{ }^{-1}\right): 3383(\mathrm{NH}), 3009$ (CH aliphatic), 1739, 1638 (C=0' s). ${ }^{1} \mathrm{H}$ NMR (300 MHz, DMSO$\left.d_{6}, \delta, \mathrm{ppm}\right): 4.9\left(\mathrm{~s}, 2 \mathrm{H}, \mathrm{CH}_{2}\right), 6.6(\mathrm{dd}, 1 \mathrm{H}, \mathrm{Hx}), 6.8\left(\mathrm{dd}, 1 \mathrm{H}, \mathrm{H}_{\mathrm{M}}\right)$, 7.7 (dd, $1 \mathrm{H}, \mathrm{H}_{\mathrm{A}}$ ), 7.2-7.9 (m, 4H, arom.), 8.09 (s, $\left.1 \mathrm{H}, \mathrm{CH}=\mathrm{N}\right)$, $11.59(\mathrm{~s}, 1 \mathrm{H}, \mathrm{NH}), 11.67$ (s, $1 \mathrm{H}, \mathrm{NH})$. Anal. calcd. for $\mathrm{C}_{15} \mathrm{H}_{12} \mathrm{~N}_{4} \mathrm{O}_{4}$ : C, 57.68; H, 3.85; N, 17.93. Found: C, 57.60; H, 3.86; N, 17.91\%.

(2,4-Dioxo-1,4-dihydro-2H-quinazolin-3-yl)-acetic acid (thiophen-2-ylmethylene)-hydrazide (6d): Yellow crystal. Yield: 0.39 g, 75\%. M.p.: > $300{ }^{\circ} \mathrm{C}$. FT-IR (KBr, v, $\left.\mathrm{cm}^{-1}\right): 3201(\mathrm{NH})$, 2924, 2852 (CH aliphatics), 1740, 1659 (C=0' s). 


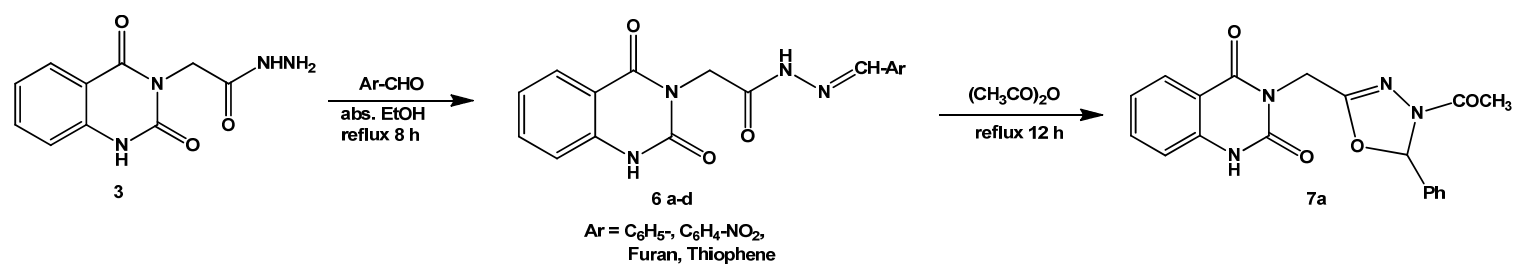

Scheme 3

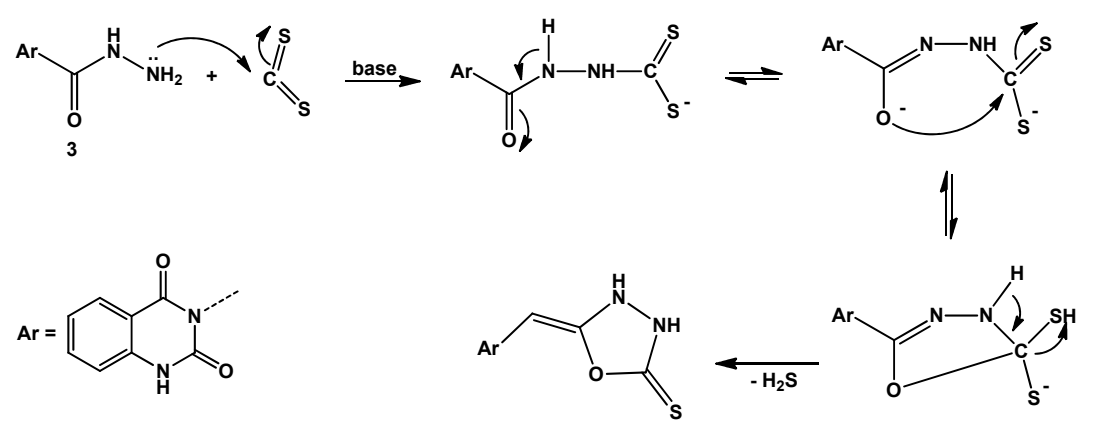

Scheme 4

${ }^{1} \mathrm{H}$ NMR (300 MHz, DMSO- $\left.d_{6}, \delta, \mathrm{ppm}\right): 4.9\left(\mathrm{~s}, 2 \mathrm{H}, \mathrm{CH}_{2}\right), 7.1$ (dd, $\left.1 \mathrm{H}, \mathrm{H}_{\mathrm{x}}\right), 7.5\left(\mathrm{dd}, 1 \mathrm{H}, \mathrm{H}_{\mathrm{M}}\right), 7.8\left(\mathrm{dd}, 1 \mathrm{H}, \mathrm{H}_{\mathrm{A}}\right), 7.2-7.9(\mathrm{~m}, 4 \mathrm{H}$, arom.), 8.4 (s, 1H, CH=N.), $11.5(\mathrm{~s}, 1 \mathrm{H}, \mathrm{NH}), 11.7(\mathrm{~s}, 1 \mathrm{H}, \mathrm{NH})$. Anal. calcd. for $\mathrm{C}_{15} \mathrm{H}_{12} \mathrm{~N}_{4} \mathrm{O}_{3} \mathrm{~S}$ : C, 54.86; H, 3.67; N, 17.05, S, 9.76. Found: C, 54.98; H, 3.80; N, 17.11, S, 9.74\%.

\subsubsection{3-(4-Acetyl-5-phenyl-4,5-dihydro-[1,3,4]oxadiazol-2- ylmethyl)-1H-quinazoline-2,4-dione (7a)}

A solution of compound $\mathbf{6 a}(0.4 \mathrm{~g}, 0.0012 \mathrm{~mol}$.) with acetic anhydride $(20 \mathrm{~mL})$ was heated under reflux for 12 hours. After cooling; the reaction mixture was poured into crushed ice to give white precipitate which collected by filtration then crystallized from ethanol to give compound $\mathbf{7 a}$ as a white crystal (Scheme 3). Yield: 0.42 g, 70\%. M.p.: > $300{ }^{\circ} \mathrm{C}$. FT-IR (KBr, v, cm $\left.{ }^{-1}\right): 3198(\mathrm{NH}), 1740,1637\left(\mathrm{C}=\mathrm{O}^{\prime} \mathrm{s}\right) .{ }^{1} \mathrm{H}$ NMR $(300$ MHz, DMSO- $\left.d_{6}, \delta, \mathrm{ppm}\right): 2.1\left(\mathrm{~s}, 3 \mathrm{H}, \mathrm{CH}_{3}\right), 4.8\left(\mathrm{~s}, 2 \mathrm{H}, \mathrm{CH}_{2}\right), 7.0$ (s, $1 \mathrm{H}, \mathrm{CH})$, 7.1-7.9 (m, 9H, arom.), $11.5(\mathrm{~s}, 1 \mathrm{H}, \mathrm{NH})$. Anal. calcd. for $\mathrm{C}_{19} \mathrm{H}_{16} \mathrm{~N}_{4} \mathrm{O}_{4}$ : C, 62.62; H, 4.40; N, 15.37. Found: C, 62.64; H, 4.42; $\mathrm{N}, 15.42 \%$.

\section{Results and discussion}

In this study, our target was to synthesize novel quinazolindiones derivatives which based on the reaction between glycine ethyl ester hydrochloride and $\mathrm{N}$ phenylsulphonyloxyphtahlimide via Lossen rearrangement [89] giving quinazoline-2,4-dione derivatives. In the course of the present work, we found, reacting of $\mathrm{N}$-phenylsulphonyl oxyphthalimide (1) with glycine ethyl ester hydrochloride in pyridine under reflux for 9 hours afford (2,4-dioxo-1,4dihydro-2H-quinazolin-3-yl)-acetic acid ethyl ester (2), which then converted to starting material (2,4-dioxo-1,4-dihydro- $2 \mathrm{H}$ quinazolin-3-yl)-acetic acid hydrazide (3) in a good yield through its reaction with hydrazine hydrate in absolute ethanol. The synthetic procedures to obtain the target compounds are depicted in Scheme 1.

Scheme 2 outlines the synthetic pathway used to obtain 3(5-thioxo-[1,3,4] oxadiazolidin-2-ylidenemethyl)- $1 H$-quinazoline-2,4-dione (4) which prepared by treatment of compound 3 with with carbon disulphide in pyridine under reflux for $9 \mathrm{hrs}$. as given mechanism in Scheme 4. Also, we can obtain 3-(4- amino-5-mercapto- $4 H$-[1,2,4] triazol-3-ylmethyl)-1 $H$-quinazoline-2,4-dione (5) by reaction of hydrazide (3) with carbon disulphide and ethanolic potassium hydroxide followed by treatment the resulted intermediate with hydrazine hydrate in distilled water.

Scheme 3 outlines our strategies for the synthesis of arylidines (6a-d) which produced by reaction of hydrazide (3) with some aromatic aldehydes like benzaldehyde, $p$ nitrobenzaldehyde, furan aldehyde and thiophenealdehyde, respectively, in ethanol. Also, we can use compound $\mathbf{6 a}$ to synthesis oxadiazole ring attached to quinazoline moiety by treatment of compound 6a with acetic anhydride under reflux for $12 \mathrm{hrs}$. afford 3-(4-acetyl-5-phenyl-4,5-dihydro-[1,3,4] oxa diazol-2-ylmethyl)-1H-quinazoline-2,4-dione (7a).

\section{Conclusion}

In conclusion, an efficient synthesis of tetrachloro quinazolindione derivatives has been developed.

\section{Acknowledgment}

The authors are grateful to Mr. Ibrahim M. A. Ismael, Central Lab. of South Valley University for his help in analysis of some samples.

\section{References}

[1]. Tiwari, A. K.; Singh, V. K.; Bajpai, A.; Shukla, G.; Singh, S.; Mishra, A. K. Eur. J. Med. Chem. 2007, 42, 1234-1238.

[2]. Grover, G.; Kini, S. G. Eur. J. Med. Chem. 2006, 41, 256-262.

[3]. Giri, R. S.; Thaker, H. M.; Giordano, T.; Williams, J.; Rogers, D.; Sudersanam, V.; Vasu, K. K. Eur. J. Med. Chem. 2009, 44, 2184-2189.

[4]. El-Helby, A.; Abdel Wahab M. Acta Pharm. 2003, 53, 127-138.

[5]. Kadi, A. A.; El-Azab, A. S.; Alafeefy, A. M.; Abdel-Hamide, S. G. Al-Azhar J. Pharm. Sci. 2006, 34, 147-158.

[6]. Jatav, V.; Mishra, P.; Kashaw, S. Eur. J. Med. Chem. 2008, 43(9), 19451951.

[7]. Zhao, L.; Tao, K.; Li, H.; Zhang, J. Tetrahedron 2011, 67(15), 2803-2806.

[8]. Hassan, M. A.; Younes, A. M. M.; Taha, M. M.; Abdel-Monsef, H. A. Eur. J Chem. 2011, 2(4), 514-518.

[9]. Hassan, A. M.; Younes, M. M. A.; Taha, M. M.; Abdel-Monsef, H. A. Chem. Sci. J. 2012, 1-12. 